\title{
Reviewing the Ethical Concerns of the Convalescent Plasma Therapy in COVID-19
}

\author{
Manish Raturi1,® Mansi Kala² Kunal Das ${ }^{3}$ Anuradha Kusum² \\ 1Department of Immunohematology and Blood Transfusion, \\ Himalayan Institute of Medical Sciences, Swami Rama Himalayan \\ University, Dehradun, Uttarakhand, India \\ 2Department of Pathology, Himalayan Institute of Medical Sciences, \\ Swami Rama Himalayan University, Dehradun, Uttarakhand, India \\ ${ }^{3}$ Department of Pediatrics; Division of Pediatric Oncology and BMT, \\ Himalayan Institute of Medical Sciences, Swami Rama Himalayan \\ University, Dehradun, Uttarakhand, India
}

\author{
Address for Correspondence Manish Raturi, Department of \\ Immunohematology and Blood Transfusion, Himalayan Institute \\ of Medical Sciences, Swami Rama Himalayan University, Swami \\ Ram Nagar, Jolly Grant Dehradun, Uttarakhand 248016, India \\ (e-mail: manishraturi@srhu.edu.in).
}

J Lab Physicians 2021;13:91-94.

\author{
Abstract \\ Keywords \\ - asymptomatic carriers \\ - ethical concerns \\ - blood donation \\ - blood safety \\ - blood transfusion \\ services \\ - convalescent plasma \\ - COVID-19 \\ - regular screening
}

The understanding of coronavirus disease 2019 (COVID-19) is evolving periodically. There is also a marked increase in COVID-19 patients' demand for the provision of COVID-19 convalescent plasma (CCP) therapy. With the review of the literature, the stage of speculation is quickly moving towards conformity, with the interim guidelines given by the Food and Drug Administration (FDA) in the United States, United Kingdom's blood transfusion services, Saudi's FDA, and the Indian Council for Medical Research, in addition to the European health authorities. It is prudent to address the ethical concerns that merit consideration while harvesting and utilizing CCP as a therapeutic tool in local hospital setting.

\section{Introduction}

In the month of December 2019, a novel coronavirus (nCoV) was reported from Wuhan, China. ${ }^{1}$ This $\mathrm{nCoV}$ is known to cause coronavirus disease 2019 (COVID-19), renamed as severe acute respiratory syndrome coronavirus 2 (SARS-CoV-2). With deaths accounting in millions, this virus quickly spread across the globe. In fact, on January 31 , 2020, the World Health Organization (WHO) declared this pandemic as a public health emergency of international concern (PHIEC). ${ }^{2}$ It may be observed in a variety of cases, from the asymptomatic carrier condition to a fully developed active respiratory illness. The COVID-19 pandemic has also affected the blood community adversely. Raturi and Kusum have documented a reduction in the number of blood donors, collections, demands, and issuances during this pandemic. ${ }^{3}$ Additionally, blood safety concerns merit a logical consideration, because they include blood donors who are without any symptoms and arrive at the blood centers as fairly healthy individuals. These symptomless, innocent blood donors (IBD), are entirely oblivious of their viral status and, therefore, the published self-assessment tools can be utilized prior to their whole blood donations (WBDs). ${ }^{4}$ These IBDs may, therefore, pose a significant health concern to not only the blood transfusion services (BTS) but also the entire blood community. While the WHO stated in 2003 that there were no transfusion-borne cases of SARS-CoV, the theoretical risk of the transfusion of this $\mathrm{nCoV}$ remains a viable possibility. The current recommendations are largely based on the recent directives from the American Association of Blood Banks (AABB), but a cautious approach is crucial now more than ever, including a rigorous donor scrutiny. ${ }^{5}$ The Food published online May 24, 2021
DOI https://doi.org/ $10.1055 / \mathrm{s}-0041-1727585$ ISSN $0974-2727$
(C2021. The Indian Association of Laboratory Physicians.

This is an open access article published by Thieme under the terms of the Creative Commons Attribution-NonDerivative-NonCommercial-License, permitting copying and reproduction so long as the original work is given appropriate credit. Contents may not be used for commercial purposes, or adapted, remixed, transformed or built upon. (https://creativecommons.org/licenses/by-nc-nd/4.0/). Thieme Medical and Scientific Publishers Pvt. Ltd. A-12, 2nd Floor, Sector 2, Noida-201301 UP, India 
and Drug Administration (FDA) has also unanimously implemented the use of the COVID-19 convalescent plasma (CCP) by apheresis from recovered patients on a case-by-case basis, provided that they fulfill the qualifying criteria for such a donation. ${ }^{6}$ When it comes to the WBD, the AABB and the FDA evaluate these individuals according to the established eligibility requirements for their donation unless they have been diagnosed with flu-like diseases. In addition, regular screening of blood donors, in combination with "mini-physical," helps deter any such symptomatic donors from giving blood. ${ }^{7} \mathrm{CCP}$, on the other hand, is harvested from recovered COVID-19 patients, which is helpful in eradicating the culprit pathogen not just from the plasma but from other tissues as well, particularly in the respiratory system. Although the use of good-quality plasma is reasonably safe, the existing global compliance with healthy donors' plasma usage according to the established recommendations is explicitly moderate to low in some developing countries. However, innumerable flaws are seen while ordering this specific blood component. Raturi et al have shown that its prophylactic use is not only doubtful but also unjustified, with the exception of clinical use, such as in coagulopathy with bleeding, prior to any invasive treatment and reversal of the effect of anticoagulants. ${ }^{8}$ In addition, CCP therapy is historically well-known to have anticipated benefits in COVID-19 patients. The recommendations in this regard have recently been revised by the U.S. FDA, UK BTS, Saudi FDA, and the Indian Council for Medical Research (ICMR), in addition to the European health authorities. ${ }^{9}$ While the interim criteria for donor eligibility have already been found by the U.S. FDA, United Kingdom BTS, Saudi FDA, ICMR, and other regional health authorities in conjunction with the process details and eligibility criteria for the recipients, these recommendations do not enlighten us much about the legal and ethical concerns that must be addressed while harvesting and using СCP as a therapeutic tool.

\section{Historical Aspects of CCP Therapy}

Historically, long before vaccines became available for most highly infectious diseases, CCP was used in the cases of polio, measles, mumps, and influenza. ${ }^{10-13} \mathrm{~A}$ meta-analysis of 1,703 patients who suffered the influenza pandemic during the 1918 Spanish flu reported a diminished mortality rate of CCP recipients. ${ }^{14}$ Conversely, in a double-blinded, randomized, placebo-controlled trial for those diagnosed with influenza A, CCP was not found to be superior to placebo. ${ }^{15}$ Moreover, 84 Ebola virus patients receiving CCP therapy without the established levels of neutralizing antibodies appeared to have little survival benefit. ${ }^{16}$ During the preceding SARS coronavirus outbreaks between 2002 and 2004, CCP was also investigated. In a study undertaken by Cheng et al, with 80 patients, it was found that patients receiving CCP had better outcomes, that is, early hospital discharge, as compared to patients receiving CCP after day 14 of the infection (15.6 vs. $58.3 \% ; p<0.001) .{ }^{17}$ Due to the lack of adequate treatment for the outbreak of COVID-19, clinical trials of CCP therapy have recently been approved by the U.S. FDA, with enhanced access under the category of single-patient emergency investigational new drug, and by the ICMR as an "off-label" therapy. ${ }^{18}$

\section{Role of Blood Transfusion Services}

\section{Addressing the Ethical Concerns of Blood Donors}

In all circumstances, the harvest of either WBD or CCP must be voluntary and nonremunerated as published in the ICMR guidelines; no coercion should be imposed on the donor, including indemnity, which is an obligation by the patient party to provide compensation (either in cash or kind) for either a WBD or a CCP donation. Besides that, the informed consent and confidentiality of the donor merit ethical consideration. Further, in compliance with the internationally established and local/regional guidelines, all processes, including apheresis, must be defined and performed appropriately. In principle, all CCP donors should qualify as regular healthy blood donors based on history (including recent recovery from COVID-19), physical examination, and rigorous testing for transfusion-transmitted infection (TTI) markers according to local guidelines. Collections and their justified use are worthy of critical evaluation through regular audits. Additionally, product wastage must be avoided by all possible means. ${ }^{19}$

\section{Facilitating Eligibility, Recruitment, and Transportation for Donors}

The U.S. FDA, United Kingdom BTS, Saudi FDA, ICMR, and other regional health authorities define the criteria for one's eligibility to be a CCP donor as follows:

- Laboratory evidence of COVID-19 disease is confirmed by either a nasopharyngeal swab and/or a positive serological test for SARS-CoV-2 antibodies.

- Complete symptom resolution 28 days before apheresis at the earliest.

Before apheresis, it is important to bear in mind that only those female donors are eligible who are nulliparous; if multiparous, they must carry laboratory evidence of the absence of human leukocyte antigen (HLA) antibodies. This decreases the recipient's risk of acquiring transfusion-related acute lung injury (TRALI) that may happen due to the presence of anti-HLA antibodies in their CCP. Therefore, both ICMR and FDA recommend testing for SARS-CoV-2 neutralizing antibody titers, if the option is available. A neutralizing antibody titer of at least 1:160 is likely to be more beneficial. Otherwise, for the estimation of antibody levels, a sample from the donated CCP should be retained later on. ${ }^{9}$ Saudi Arabian national collaborative study opted for an interesting strategy of utilizing a rapid antibody screening using a handy lateral flow cartridge method to mandate evidence of anti-SARS-CoV2 IgG antibodies in prospective CCP donors and saved aliquots of donated CCP for later testing when this facility would widely be available in the country. ${ }^{20}$ Further, in other studies, CCP donors were selected based on a predefined minimum neutralizing antibodies titer only. ${ }^{21,22}$ Besides, to 
assess their eligibility for blood donation, CCP donors may even be encouraged to travel to the nearest blood center. Conveyances could also be provided, if needed, to facilitate travel for the same intention.

\section{Safety Assessments and Adverse Event Reporting}

- Any unfavorable event that occurs in a patient receiving regular donor plasma or CCP therapy is an adverse event (AE). ${ }^{23,24}$ In general, it is possible to further identify these as:

- AEs related to donors: These events are differentiated into local and systemic reactions. Based on their intensity, they can also be classified as mild, moderate, or severe. Hypotensive reactions, citrate reactions, hematomas, loss of consciousness, seizures, and allergic reactions may further be described in an etiology-based classification.

- Equipment- and/or kit-related AEs: These events are secondary to disposable sets that are defective or erroneous. They can lead to hemolysis, formation of thrombosis, air embolism, leakage, infection due to improper equipment installations, and so on.

- Recipient-based AEs: A transfusion-related AE in a recipient is a consequence that is momentarily related to the administration of blood or blood components. TRALI, transfusion-related circulatory overload, allergic/anaphylactic reactions, TTI transmission, and febrile nonhemolytic transfusion events make up the majority of semilethal and medically curable AEs generally associated with plasma transfusions.

\section{Addressing the Ethical Concerns of Recipients}

Before any blood or blood component transfusion, recipients are entitled to receive adequate and legible information on the associated risks of plasma transfusions, particularly the AEs. If any extra steps have been taken to mitigate the risk of TTI (for example, pathogen reduction treatment), it should be mentioned in the informed consent. Their informed consent forms must be kept safely, following local hospital transfusion policies. ${ }^{19}$ Signed documents must then be collected and kept in the medical records of the patients, and the doctors must ensure that they do so regularly. Besides that, all paper and computer data entered in the recipient's medical record must be kept confidential and accessible to authorized personnel only. These confidential records and data can be stored under the properly specified disposition policies at the local hospital facilities. Although the relevant country/regional guidelines do mention recommendations toward one's candidature for CCP therapy, they do not provide a decision-making benchmark as to who should be the first to start receiving СCP therapy. They leave it entirely up to the clinicians and hospitals to decide who would need it the most. This merits ethical consideration on the part of the treating physician and the hospitals collaboratively. The best approach may be to have a multidisciplinary team make a decision. To resolve this ethical problem, hospitals may opt to use a predetermined randomization model, such that the decision does not fall within the scope of their individual preference. Another resolution could be the use of CCP therapy in recipients on a first-come-and-first-served basis. The hospitalization costs incurred and the facility for availing medical insurance claims also need to be critically analyzed.

\section{Conclusion}

In conclusion, the understanding of COVID-19 is evolving periodically. In fact, ethical concerns about priorities would ease as more people are tested, treated, and come forward to donate their CCP for their local as well as community needs. Given our limited experience with $\mathrm{nCoV}$, we expect additional changes to the interim guidelines in an attempt to adapt further. In fact, we are still at a speculation stage and an attempt to comply with these interim guidelines along with an assessment of the latest available literature could, in principle, help to address all ethical concerns when harvesting and using $\mathrm{CCP}$ as a therapeutic tool for patients.

\section{Financial Support and Sponsorship}

None.

\section{Informed Consent}

As per the department policy, an informed consent is obtained from all donors prior to blood donation in accordance with our department protocol.

\section{Ethical Approval}

All procedures performed in studies involving human participants were in accordance with the ethical standards of the Institutional Ethics Committee and with the 1964 Helsinki Declaration and its later amendments or comparable ethical standards. The article does not contain any regulated human participant or animal research.

\section{Funding and Resources}

No funding was received for this study by any entity in the public or private sector.

\section{Authors' Contribution List}

Manish Raturi and Mansi Kala designed the concept. Manish Raturi and Kunal Das did the literature search. Manish Raturi compiled the data. Manish Raturi, Mansi Kala, Kunal Das, and Anuradha Kusum prepared and edited the manuscript. Manish Raturi, Mansi Kala, Kunal Das, and Anuradha Kusum reviewed the manuscript. Manish Raturi, as a guarantor, takes the responsibility of the integrity of the work, as a whole, right from its inception to being a published article.

\section{Conflict of Interest}

None declared. 


\section{References}

1 Sahu KK, Mishra AK, Raturi M, Lal A. Current perspectives of convalescent plasma therapy in COVID-19. Acta Biomed 2020;91(4):e2020175

2 World Health Organization rolling updates on coronavirus disease (COVID-19);2020. Available at: https://www.who. int/emergencies/diseases/novel-coronavirus-2019/events-asthey-happen. Accessed March 17, 2020

3 Raturi M, Kusum A. The blood supply management amid the COVID-19 outbreak. Transfus Clin Biol 2020;27(3):147-151

4 Raturi M. A quick "can I donate blood" self-assessment tool amid the COVID-19 outbreak. Transfus Clin Biol 2020; 27(3):169-170

5 American Association of Blood Banks (AABB)'s Coronavirus Resources;2020. Available at: http://www.aabb.org/advocacy/ regulatorygovernment/Documents/Impact-of-2019-NovelCoronavirus-on-Blood-Donation.pdf. Accessed March 18, 2020

6 American Society of Hematology. Clinical news: online exclusives. Available at: https://www.ashclinicalnews.org/ online-exclusives/fda-allows-blood-plasma-treatment-coronavirus-case-case-basis/. Accessed April 07, 2020

7 Raturi M, Kusum A. The active role of a blood center in outpacing the transfusion transmission of COVID-19. Transfus Clin Biol 2020;27(2):96-97

8 Raturi M, Shastry S, Murugesan M, Baliga PB, Chakravarthy K. Effect of plasma component transfusion on conventional coagulation screening tests. Asian J Transfus Sci 2018;12(1):57-61

9 Directorate General Health Services, Government of India. Clinical trial of convalescent plasma in COVID-19 patients. [17.04.2020] Available at: https://cdsco.gov.in/opencms/ opencms/system/modules/CDSCO.WEB/elements/download_ file_division.jsp?num_id=NTg2Mw==. Accessed May 29, 2020

10 Park WH. Therapeutic use of antipoliomyelitis serum in preparalytic cases of poliomyelitis. JAMA 1932;99(13): 1050-1053

11 Luke TC, Casadevall A, Watowich SJ, Hoffman SL, Beigel JH, Burgess TH. Hark back: passive immunotherapy for influenza and other serious infections. Crit Care Med 2010;38suppl. 4:e66-e73

12 Rambar AC. Mumps: use of convalescent serum in the treatment and prophylaxis of orchitis. Am J Dis Child 1946;71:1-13

13 Gallagher JR. Use of convalescent measles serum to control measles in a preparatory school. Am J Public Health Nations Health 1935;25(5):595-598
14 Luke TC, Kilbane EM, Jackson JL, Hoffman SL. Metaanalysis: convalescent blood products for Spanish influenza pneumonia: a future H5N1 treatment? Ann Intern Med 2006;145(8):599-609

15 Beigel JH, Aga E, Elie-Turenne M-C, et al; IRC005 Study Team. Anti-influenza immune plasma for the treatment of patients with severe influenza A: a randomised, double-blind, phase 3 trial. Lancet Respir Med 2019;7(11):941-950

16 van Griensven J, Edwards T, de Lamballerie X, et al; Ebola-Tx Consortium. Evaluation of convalescent plasma for Ebola virus disease in guinea. $\mathrm{N}$ Engl J Med 2016;374(1):33-42

17 Cheng Y, Wong R, Soo YO, et al. Use of convalescent plasma therapy in SARS patients in Hong Kong. Eur J Clin Microbiol Infect Dis 2005;24(1):44-46

18 US Food and Drug Administration. Recommendations for Investigational COVID-19 convalescent plasma. FDA. 2020. Available at: https://www.fda.gov/vaccines-blood-biologics/ investigational-new-drug-ind-or-device-exemption-ide-process-cber/recommendations-investigational-covid-19-convalescent-plasma. Accessed June 24, 2020

19 Netherlands: International Society of Blood Transfusion. The ISBT code of ethics. Available at: http://www.isbtweb.org/ fileadmin/user_upload/_About_ISBT/ISBT_Code_of_Ethics_ English.pdf. Accessed February 4, 2021

20 Albalawi M, Zaidi SZA, AlShehry N, et al. Safety and efficacy of convalescent plasma to treat severe COVID-19: protocol for the Saudi collaborative multicenter phase II study. JMIR Res Protoc 2020;9(10):e23543

21 Duan K, Liu B, Li C, et al. Effectiveness of convalescent plasma therapy in severe COVID-19 patients. Proc Natl Acad Sci U S A 2020;117(17):9490-9496

22 Shen C, Wang Z, Zhao F, et al. Treatment of 5 critically ill patients with COVID-19 with convalescent plasma.JAMA 2020; 323(16):1582-1589

23 ISBT/IHN. ISBT/IHN 2014 definitions for complications related to blood donation. Standard for Surveillance of Complications Related to Blood Donation. Available at: https://www.isbtweb. org/fileadmin/user_upload/Donor_Standard_Definitions_ Final_2014.pdf. Accessed June 23, 2020

24 Pandey S, Vyas GN. Adverse effects of plasma transfusion. TRANSFUSION 2012;52(Suppl 1):65S-79S 